\title{
Paraneoplastic neurological syndrome and polyglandular autoimmune syndrome type 2 in a case of small cell lung cancer
}

\author{
S Watanabe, ${ }^{1}$ J Tanaka, ${ }^{1}$ T Ohta, ${ }^{1}$ H Yoshizawa, ${ }^{2}$ F Gejyo
}

\begin{abstract}
${ }^{1}$ Department of Medicine (II), Niigata University Medical and Dental Hospital, Niigata-city, Niigata, Japan; ${ }^{2}$ Bioscience Medical Research Centre, Niigata University Medical and Dental Hospital, Niigata-city, Niigata, Japan

Correspondence to: Dr J Tanaka, Department of Medicine (II), Niigata University Medical and Dental Hospital, 1-754 Asahimachi-dori, Chuo-ku, Niigata-city, Niigata 951-8520, Japan; juntatnk@

med.niigata-u.ac.jp
\end{abstract}

Received 5 December 2007 Accepted 19 February 2008

\section{ABSTRACT}

We report a female patient with small cell lung cancer (SCLC) and clinical findings consistent with polyglandular autoimmune syndrome type 2 (PGA2) and paraneoplastic neurological syndrome (PNS). To the best of our knowledge, this is the first reported case of SCLC associated with PGA2 and PNS. All of the autoantibodies detected before anticancer treatment decreased below the upper normal limits after serial treatment, and the patient's clinical symptoms also improved. Cross reactivity of autoantibodies may have contributed to the complicated clinical picture of this patient.

\section{CASE REPORT}

A 71-year-old woman was admitted with ataxic gait, numbness in all of her fingers and toes which had developed gradually over the previous 5 months. She had a 7.5 pack-year smoking history and a 5 year history of diabetes which was managed well with dietary therapy. No family history of endocrinological disorders had been documented. ECOG Performance Status (PS) was grade 1 .

Neurological examination showed a marked decrease in sensation to all modalities in the glove and stocking distribution with loss of deep tendon reflexes. Significant ataxia of stance, gait and limbs was also present. Laboratory data revealed a fasting blood glucose value of $20.5 \mathrm{mmol} / 1$ (normal 3.96.0) and glycated haemoglobin A1C (HbA1c) of $10.2 \%$ (normal 4.3-5.8). CSF contained 6 mononuclear cells $/ \mathrm{mm}^{3}$ (normal 0-5) with a glucose level of $6.34 \mathrm{mmol} / \mathrm{l}$ (normal 2.22-3.89) and a protein level of $2.24 \mathrm{~g} / 1$ (normal 0.15-0.5).

A chest CT scan demonstrated a mass measuring $38 \times 31 \mathrm{~mm}$ in the right lower lobe and right hilar lymphadenopathy. Bronchoscopy revealed endobronchial stenosis with mucosal elevation in the right lower branch where biopsy specimens were obtained. An abdominal CT, a radionuclide bone scan and a cerebral MRI scan showed no abnormalities. The final oncological diagnosis was small cell carcinoma, limited disease.

Endocrinological studies revealed autoimmune thyroiditis with positive antithyroid peroxidase (TPO) and antithyroglobulin (Tg) antibodies. Antibodies to glutamic acid decarboxylase (GAD) were also positive. Human leucocyte antigen typing detected the allele DR4.

Although no reactivity of serum antineuronal antibody was found by ELISA, the patient's serum reacted with Purkinje cells of mouse cerebellum on immunohistological study. Western blotting showed reactivity against homogenates of mouse cerebellum, human cerebral cortex and human subcortical neurons at a molecular weight of $150 \mathrm{kDa}$ (fig 1).

The patient was treated with four cycles of chemotherapy with day 1 carboplatin at an AUC of $5.0 \mathrm{mg} / \mathrm{ml} / \mathrm{min}$, and day $1-3$ etoposide at $80 \mathrm{mg} / \mathrm{m}^{2}$ administered every 3 weeks, followed by sequential thoracic radiation therapy (6000 cGy). Intensive insulin therapy was started at $0.6 \mathrm{U} / \mathrm{kg} /$ day, and no corticosteroids were administered to minimise hyperglycaemia. She received two trials of intravenous immune globulin at $400 \mathrm{mg} / \mathrm{kg} /$ day for 5 days before and during treatment.

After serial treatment, a chest CT showed complete disappearance of the pulmonary mass, and her neurological symptoms improved partially. HbA1c was restored to normal, and insulin requirements decreased to $0.2 \mathrm{U} / \mathrm{kg} /$ day. CSF contained 1 mononuclear cell $/ \mathrm{mm}^{3}$ and protein level was $1.07 \mathrm{~g} / \mathrm{l}$. Anti-TPO, anti-Tg and antiGAD antibodies decreased below upper normal limits. She achieved a complete response (CR) and went on to receive prophylactic cranial irradiation (3000 cGy).

Ten months after achieving CR, the gait disturbance developed again. Thoracic CT and cerebral MRI showed that the cancer relapsed as pulmonary and brain metastases. Considering her age and impaired PS of grade 2 , she did not receive secondline chemotherapy. Although no anticancer agent was administered after recurrence, she maintained her PS for another 7 months with slow disease progression. Twenty-six months after diagnosis of small cell lung cancer (SCLC), she died of a urinary tract infection.

\section{DISCUSSION}

Most or all cases of paraneoplastic neurological syndrome (PNS) are believed to be immune mediated, and a variety of autoantibodies have been characterised in PNS. ${ }^{1}$ Anti-GAD antibodies are not only described in type 1 diabetes, but also in neurological diseases with proven or suspected autoimmune origin, such as Lambert-Eaton myasthenic syndrome, stiff person syndrome and chronic cerebellar syndrome, because GAD is present in pancreatic islet beta cells and neurons. ${ }^{2-5}$ Our patient's blood glucose level was well controlled before the onset of PNS but was elevated on admission. As the serum test for antiGAD antibodies was positive, we speculated that 
Antineuronal antibodies

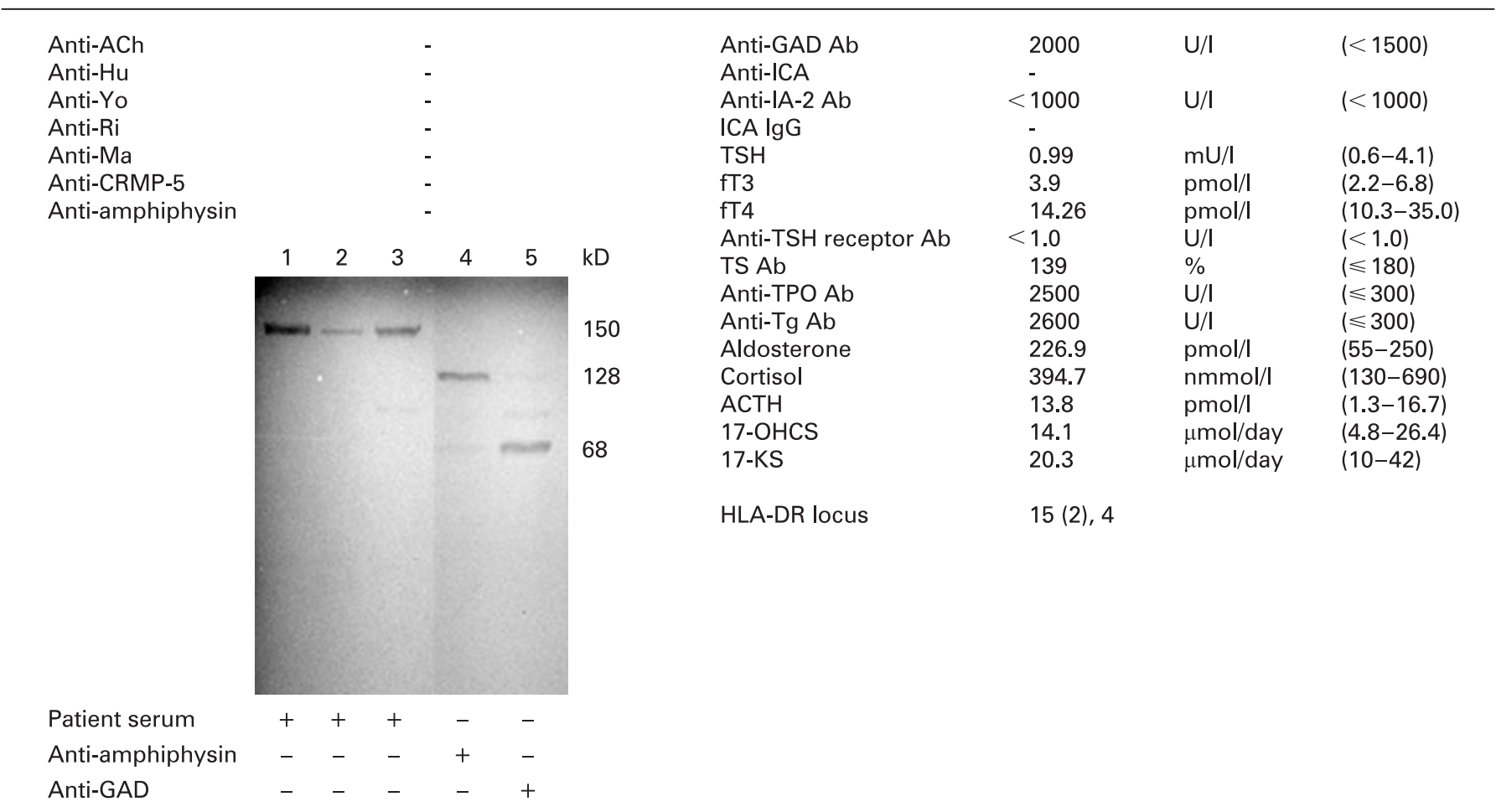

Figure 1 Summary of autoimmune antibodies and basal hormone levels. Western blot using the patient's serum (1:200) with human cerebral grey matter homogenate (lane 1), human cerebral white matter homogenate (lane 2) and mouse cerebellum homogenate (lane 3). As control, antiamphiphysin antibody (lane 4, 1:1000) and anti-GAD antibody (lane 5, 1:1000) were used with human cerebral grey matter homogenate. The patient's serum reacted to human cerebral grey matter, human cerebral white matter and mouse cerebellum at a molecular weight of $150 \mathrm{kDa}$. 17-KS, 17ketosteroids; 17-OHCS, 17-hydroxycorticosteroids; Ab, antibody; ACh, acetylcholine; ACTH, adrenocorticotropic hormone; CRMP-5, collapsin response mediated protein 5; fT3, free triiodothyronine; fT4, free thyroxine; GAD, glutamic acid decarboxylase; HLA, human leucocyte antigens; ICA, islet cell antibodies; TSH, thyroid stimulating hormone; Tg, thyroglobulin; TPO, thyroid peroxidase.

anti-GAD antibodies were associated with both hyperglycaemia and neurological symptoms. Moreover, western blotting for mouse cerebellar Purkinje cells, human cortical neurons and human subcortical neurons revealed that the serum of our patient contained antineuronal antibodies bound to protein at $150 \mathrm{kDa}$. There was a possibility that these unknown antineuronal antibodies were also associated with her neurological disorders.

Extensive laboratory studies revealed that our patient had diagnostic features of polyglandular autoimmune syndrome type 2 (PGA2) (autoimmune thyroiditis, type 1 diabetes, adult woman, HLA-DR4), also known as Schmidt syndrome. Although there has been a reported correlation of cerebellar ataxia with PGA2, it is extremely rare that SCLC, paraneoplastic cerebellar degeneration, paraneoplastic sensory neuropathy and PGA2 are observed in one patient. ${ }^{5,6}$ After chemoradiotherapy, our patient achieved CR, and all of her autoantibodies were decreased. Her blood glucose and HbA1c were restored to normal. Her neurological symptoms improved, and examination of CSF showed almost normal findings. Taken together, it seemed that the autoantibodies found in our patient's serum cross reacted with her SCLC, nervous system and pancreatic islet cells.

Patients with PNS tend to have a better prognosis, and antitumour immune responses have been documented in PNS. ${ }^{17-9}$ In our case, the patient survived for 12 months after recurrence of pulmonary and brain metastases under the best supportive care.

To our knowledge, this is the first case reported of a patient with SCLC presenting with PNS and PGA2. Autoantibodies may have played a critical role in this patient's clinical complication and fairly long survival.

Competing interests: None declared.

Ethics approval: Ethics approval was obtained.

Patient consent: Obtained from patient's next of kin.

\section{REFERENCES}

1. Darnell RB, Posner JB. Paraneoplastic syndromes involving the nervous system. N Engl J Med 2003;349:1543-54

2. Solimena M, Foli F, Denis-Donini $S$, et al. Autoantibodies to glutamic acid decarboxylase in a patient with stiff-man syndrome, epilepsy, and type I diabetes mellitus. N Engl J Med 1988;318:1012-20.

3. Hermitte L, Martin-Moutot N, Boucraut J, et al. Humoral immunity against glutamic acid decarboxylase and tyrosine phospatase IA-2 in Lambert-Eaton myasthenic syndrome. J Clin Immunol 2000;20:287-93.

4. Baekkeskov S, Anastoot HJ, Christgau S, et al. Identification of the 64K autoantigen in insulin-dependent diabetes as the GABA-synthesizing enzyme glutamic acid decarboxylase. Nature 1990:347:151-6.

5. Honnorat J, Saiz A, Giometto B, et al. Cerebellar ataxia with anti-glutamic acid decarboxylase antibodies. Arch Neurol 2001;58:225-30.

6. Seissler J, Bieg S, Yassin N, et al. Association between antibodies to the MR 67,000 isoform of glutamate decarboxylase (GAD) and type 1 (insulin-dependent) diabetes mellitus with coexisting autoimmune polyendocrine syndrome type2. Autoimmunity 1994;37:61-9

7. Graus F, Dalmau J, Rene M, et al. Anti-Hu antibodies in patients with small-cell lung cancer: association with complete response to therapy and improved survival. J Clin Oncol 1997;15:2866-72.

8. Maddison P, Newsom-Davis J, Mills KR, et al. Favourable prognosis in LambertEaton myasthenic syndrome and small-cell lung carcinoma. Lancet 1999;335:117-18.

9. Rojas I, Graus F, Keime-Guibert F, et al. Long-term clinical outcome of paraneoplastic cerebellar degeneration and anti-Yo antibodies. Neurology 2000;55:713-15. 